Background Efficient matching of energy supply to demand is essential for maintaining normal cardiac function. Altered cardiac metabolism may contribute to the development of cardiac dysfunction by impairing metabolic flexibility in type 2 diabetes (T2D). Utilising simultaneous coronary sinus (CS) and aorta blood sampling and cardiac magnetic resonance imaging (CMR), we aimed to evaluate the effect of T2D on myocardial substrate preferences in response to acute increases in cardiac workload and the effects on contractile function.

Methods Eligible participants without obstructive coronary artery disease $(>50 \%$ luminal coronary artery stenosis on coronary angiography) underwent transmyocardial arteriovenous blood sampling. Metabolites in paired coronary sinus and arterial samples were quantified to determine myocardial fuel selection at rest and during a stress protocol with intravenous dobutamine. Fatty acid (FA), glucose, 3-hydroxybutyric acid (3HBA) and lactate utilisation at rest and haemodynamic stress was calculated as an extraction fraction \% (EF). Participants underwent dobutamine stress multiparametric CMR imaging at 3.0 Tesla (Skyra, Siemens, Germany) on a separate visit within 21 days to quantify cardiac volumes, function and perfusion.

Results Two thousand and sixty-one participants were screened and due to stringent inclusion and exclusion criteria for this mechanistic study, three T2D patients and five matching controls were enrolled. Baseline demographics and glycometabolic

Abstract 125 Table 1 Baseline demographics and glycometabolic data

\begin{tabular}{lll}
\hline & T2D $(\mathbf{n}=3)$ & Controls $(\mathrm{n}=5)$ \\
\hline Age (yr) & $60 \pm 2$ & $59 \pm 15$ \\
Gender (M) & 3 & 4 \\
BMI (kg/m2) & $29.8 \pm 7.1$ & $31.3 \pm 5.8$ \\
Weight (kg) & $85.4 \pm 24.3$ & $91.0 \pm 22.9$ \\
Systolic BP (mmHg) & $121 \pm 7$ & $129 \pm 10$ \\
Diastolic BP (mmHg) & $78 \pm 2$ & $79 \pm 14$ \\
Baseline Heart Rate (bpm) & $57 \pm 10$ & $62 \pm 5$ \\
Peak Stress Heart Rate (bpm) & $120 \pm 7$ & 11614 \\
Fasting glucose (mmol/L) & $7.1 \pm 2.4$ & $5.1 \pm 0.6$ \\
HOMA IR & $14.1 \pm 9.7$ & $3.9 \pm 1.6$ \\
Total Cholesterol: HDL & $3.0 \pm 0.2$ & $3.0 \pm 0.8$ \\
HbA1C (mmol/mol) & $52 \pm 19$ & $37 \pm 2$ \\
\hline
\end{tabular}

Abstract 125 Table 2 Echocardiographic and CMR Data

\begin{tabular}{lll}
\hline & T2D $(n=3)$ & Controls $(n=5)$ \\
\hline Echocardiographic & & \\
Data & & \\
E/A & $0.99 \pm 0.28$ & $0.92 \pm 0.20$ \\
Average E/E' & $15.17 \pm 7.73$ & $8.50 \pm 3.84$ \\
CMR Data & & \\
LV EDVi (ml/m2) & $101.5 \pm 43.8$ & $82.6 \pm 8.9$ \\
LVEF (\%) & $53.0 \pm 21.7$ & $59.9 \pm 4.1$ \\
LV Mass Index (g/ & $66.0 \pm 23.0$ & $63.7 \pm 13.0$ \\
m2) & & \\
LV Mass/Nolume & $0.66 \pm 0.05$ & $0.76 \pm 0.09$ \\
(g/ml) & & \\
\hline
\end{tabular}

LV EDV: Left Ventricular End Diastolic Volume Index; LVEF: Left Ventricular Ejection
Mean Extraction Fraction of 3 Hydroxybutyric Acid (3-HBA) Rest vs Stress

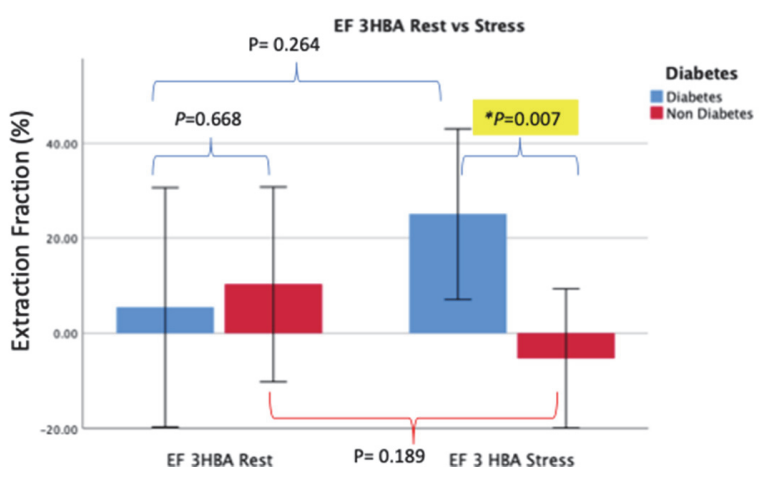

Abstract 125 Figure 1

results are documented in table 1. Mean 3-HBA changes calculated as extraction fraction at stress and rest are illustrated in figure 1. Table 2 shows the major echocardiographic and CMR results. There were no significant differences in FA or glucose uptake between T2D patients and controls at rest or stress. 3HBA EF was significantly increased in T2D during stress $(25.04 \%$ vs $-5.31 \%, \mathrm{p}=0.007)$.

Conclusions We demonstrate for the first time in vivo that the diabetic heart switches to ketone bodies during increased workloads as a significant fuel source. At present, it is unknown whether enhanced ketone body metabolism in T2D is beneficial, maladaptive, or a bystander. As the energetic properties of ketones are favourable, increased myocardial ketone oxidation could be an adaptive change designed to compensate for defects in myocardial energy metabolism in diabetes. Further, larger studies are warranted.Funding acknowledgement: This research was funded by the British Heart Foundation Clinical Research Training Fellowship and the Wellcome Trust on a Seed Grant

Conflict of Interest None

\section{IS HEART FAILURE ADMISSION OVER WEEKENDS AND BANK HOLIDAYS ASSOCIATED WITH HIGHER RISK OF DEATH?}

${ }^{1}$ Reham Awad, ${ }^{1}$ Prince Joseph, 'Emily Owen, 'Shamshad Khan, ${ }^{1}$ Mohammed Hilal-Babu, ${ }^{1}$ Sunita Avinash, 'Sundas Masudi, 'Jameela Bahar, ${ }^{1}$ Haydn Bennett, ${ }^{2}$ Kenneth Wong, ${ }^{1}$ Amna Rahman. 'Blackpool Teaching Hospitals NHS Foundation Trust (Blackpool Victoria Hospital), Blackpool, UK; ${ }^{2}$ Blackpool Teaching Hospitals

\subsection{6/heartjnl-2021-BCS.123}

Introduction - Acute Heart failure inpatient mortality is as high as $7-11 \%$ in UK National Heart Failure audits with little improvement over many years. Some but not all studies have suggested a possible weekend effect on mortality in different patient groups including patients suffering from heart failure. However, some studies failed to recognise admissions after $5 \mathrm{pm}$ on Fridays as weekend. We aim to determine in a large cohort of heart failure admissions whether patients are less likely to survive if admitted during weekend or Bank Holidays.

Methods - A retrospective study of consecutive admissions with heart failure as 1st diagnostic position in Blackpool Victoria Hospital over an 18 month period from 1st August 
2019 to 31st January 2021. Normal working hours is defined as 9 am to $5 \mathrm{pm}$ during weekdays except UK Bank holidays.

Results - Of 1178 episodes of heart failure admission, there were 140 inpatient deaths (11.9\%). Median age at admission was 80 [interquartile range 70, 86]. 48 (4.1\%) were COVID positive, $34 \%$ negative. $60.4 \%$ were not tested. 405 patients were admitted over the weekend/bank holiday (34.4\%). The other 773 patients (65.6\%) were admitted during normal working hours ( $9 \mathrm{am}$ to $5 \mathrm{pm}$ during weekdays). The length of stay is not significantly different whether patients are admitted over weekend /bank holidays or during normal working hours \{median 11 days [5, 19] vs 11 days [5,21] admitted during normal working hours, $\mathrm{p}=0.48\}$. Inpatient mortality in patients admitted with heart failure over the weekend/bank holiday was 1.3 times higher, but this apparent increased risk was not statistically significant $(95 \%$ confidence interval of the odds ratio $=0.98-1.8$, Fisher exact 2-tailed $\mathrm{p}=0.2$ ).

Conclusion There is no significant difference in inpatient mortality between patients admitted with heart failure during normal working hours or during weekends and bank holidays. Future research will explore reasons (including the use of evidence based drugs, timely diagnosis with echocardiography and the impact of COVID).

Conflict of Interest Heart Failure

\section{RESPONSE TO CARDIAC RESYNCHRONISATION THERAPY IS ASSOCIATED WITH REDUCTION IN SERUM PARATHYROID HORMONE LEVEL}

${ }^{1}$ Mark Mills, ${ }^{2}$ Paul Sheridan, ${ }^{3}$ Patricia Lawford, ${ }^{1}$ Abdallah Al-Mohammad, ${ }^{4}$ David Warriner. ${ }^{1}$ Sheffield Teaching Hospitals NHS Foundation Trust, Sheffield, UK; ${ }^{2}$ Chesterfield Royal Hospital; ${ }^{3}$ University of Sheffield; ${ }^{4}$ Doncaster and Bassetlaw Teaching Hospitals NHS Foundation Trust

\subsection{6/heartjnl-2021-BCS.124}

Introduction In patients with heart failure with reduced ejection fraction ( $\mathrm{HFrEF})$, a raised serum parathyroid hormone (PTH) level is associated with increased morbidity and mortality. Previous studies suggest that PTH levels correlate with peak VO2 and markers of endothelial dysfunction in HFrEF. We examined the role of PTH in the pathophysiological difference between response and non-response to cardiac resynchronisation therapy (CRT) in HFrEF.
Methods All patients met National Institute for Health and Care Excellence criteria for CRT implantation and were prospectively recruited from a single centre. A positive response to CRT was defined as an improvement in all four of the following domains: 1) a greater than $1 \mathrm{ml} / \mathrm{kg} / \mathrm{min}$ increase in peak $\mathrm{VO} 2$; 2) a $10 \%$ reduction in left ventricular end-systolic volume (LVESV); 3) a $10 \%$ reduction in symptoms as measured by the Minnesota living with heart failure questionnaire (MLWHFQ); 4); over 10\% increase in 6 minute walk distance (6MWD). PTH levels were measured at baseline and at 6 months following CRT. Percentage change in PTH level was calculated as (6 month PTH level - baseline PTH level)/(baseline PTH level). Data are presented as mean \pm standard error of the mean. Analyses were performed using IBM SPSS version 24. Paired continuous data were compared with paired $t$ test; unpaired continuous data with independent sample $t$ test. Tests were 2-tailed. $\mathrm{p}<0.05$ was considered statistically significant.

Results A total of 19 patients were studied (9 responders, 10 non-responders; 95\% male, age $70 \pm 1.9$ years). At baseline, there was no significant difference in estimated glomerular filtration rate $\left(64.1 \pm 3.2 \mathrm{ml} / \mathrm{min} / 1.73 \mathrm{~m}^{2}\right.$ in responders, $58.3 \pm$ $3.9 \mathrm{ml} / \mathrm{min} / 1.73 \mathrm{~m}^{2}$ in non-responders; $\left.\mathrm{p}=0.27\right)$, corrected serum calcium level $(2.31 \pm 0.02 \mathrm{mg} / \mathrm{dL}$ in responders, 2.36 $\pm 0.02 \mathrm{mg} / \mathrm{dL}$ in non-responders; $\mathrm{p}=0.13$ ), or serum 25 hydroxy vitamin D levels $(56.5 \pm 6.8 \mathrm{ng} / \mathrm{mL}$ in responders and $45.1 \pm 12 \mathrm{ng} / \mathrm{mL}$ in non-responders; $\mathrm{p}=0.44$ ) in between the two groups. At baseline, PTH levels in responders were $85.4 \pm 9.9 \mathrm{pg} / \mathrm{mL}$, compared to $63.4 \pm 9.8 \mathrm{pg} / \mathrm{mL}$ in nonresponders $(\mathrm{p}=0.06)$. At 6 months, PTH levels in responders were $72.3 \pm 11.5 \mathrm{pg} / \mathrm{mL}$, compared to $87.8 \pm 10.7 \mathrm{pg} / \mathrm{mL}$ in non-responders $(\mathrm{p}=0.2)$. There was a significant difference in the percentage change in PTH levels between responders (an average reduction of $16 \pm 3.6 \%$ ) and non-responders (an average increase of $38 \pm 3.4 \%)(\mathrm{p}=0.002)$ (figure 1A). All responders had a decrease in serum PTH level at 6 months, whereas 8 out of 10 non-responders had an increase in serum PTH levels at 6 months (figure 1B).

Conclusion This small, single-centre study suggests that a positive response to CRT is associated with a reduction in serum PTH levels at 6 months following the procedure. Conversely, non-response to CRT is generally characterised by an increase in PTH levels at 6 months. The pathophysiological role of PTH in CRT response warrants further investigation. Conflict of Interest Nil.
A

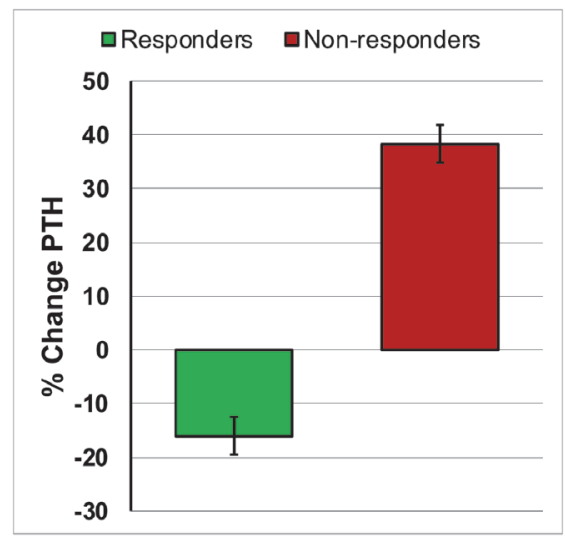

B

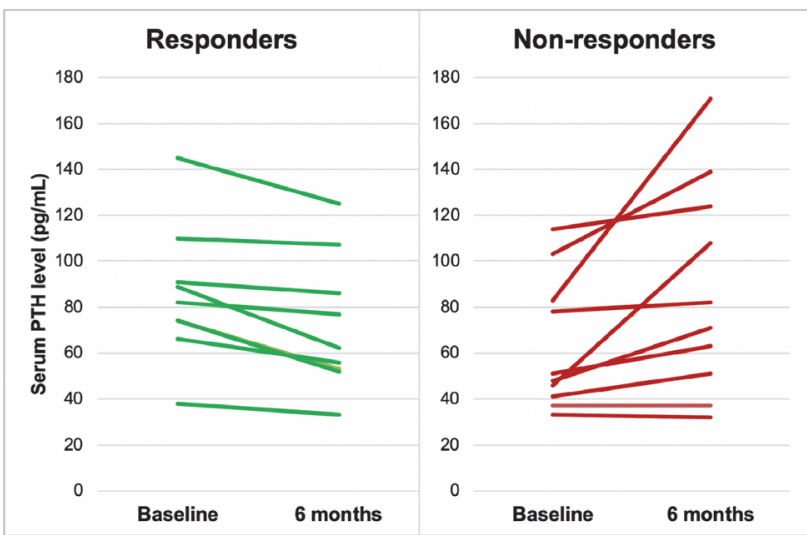

